\title{
The Sense of Gain From the Perspective of Psychology
}

\author{
Zhang Minmin ${ }^{1},{ }^{\mathrm{a}}, \mathrm{Hu} \mathrm{\textrm {Yu } ^ { 2 } , { } ^ { * }}$ \\ ${ }^{1}$ Institute of Psychology and Behavior, Wenzhou University, Wenzhou, Zhejiang, China \\ a243575652@qq.com \\ b*psyhuyu@126.com
}

\begin{abstract}
Sense of gain is a new word with both psychological connotation and characteristics of the times. The idea of sense of gain is the embodiment of the idea of "people-centered", and also reflects the people's pursuit of a better life. This paper introduces the origin and meaning of the sense of acquisition from the perspective of psychology, combs the relevant research of the sense of acquisition, analyzes various factors that affect the sense of acquisition, and discusses the direction of future research, aiming to provide a new perspective for future research.
\end{abstract}

Keywords: Sense of gain, psychological experience, cognitive experience

\section{心理学视野下的获得感}

\author{
张敏敏 ${ }^{1,}$, , 胡瑜 ${ }^{1,}$, b* \\ '温州大学心理与行为研究所, 温州, 浙江, 中国 \\ a243575652@qq.com \\ b*psyhuyu@126.com
}

\section{摘要}

获得感是一个既具有心理内涵又赋予时代特色的新词，获得感的提出是 “以人民为中心” 思想的体现， 更体现了人民对于美好生活的追求。本文从心理学视野介绍了获得感的由来、涵义,梳理了获得感的相 关研究, 分析了影响获得感的多种因素, 并探讨了今后研究的方向, 旨在为今后的研究提供新的视角。 关键词: 获得感, 心理体验, 认知体验

\section{1. 前言}

在党的十九大报告上, 习近平总书记强调, 要 “保 证全体人民在共建共享发展中有更多获得感, 不断促进 人的全面发展、全体人民共同富裕。” “使人民获得感、 幸福感、安全感, 更加充实、更有保障、更可持续。”

“获得感” 近年来已经成为与 “幸福感”、“安全感”

“满意度” 并述的心理词汇, 这其中 “幸福感”、“安 全感” 与 “满意度” 作为常用变量经常被用来评估人 们的需求满足程度与生活体验的状况, 那么什么是获得 感呢?

获得感一词自提出以来, 就得到了社会各界广泛关 注, 获得感本身就是关系到社会和人民福祉的重要关键 词, 更是由于它的 “接地气” 而深入到广大人民群众的 内心。获得感对社会和个体的发展都起着重要的作用, 然而目前与获得感相关的研究可谓是风毛麟角, 心理学 界对获得感的研究也是刚刚起步, 研究的成果也较为繁 杂并存在一定的争论。因此, 本文就目前获得感的研究 作一个简要的梳理, 希望能为今后的研究起到抛砖引玉 的作用。

\section{2. 获得感概念界定}

“获得感” 是什么？从字面层面上来讲, “获得感” 是由 “获得” 和 “感” 组成, 有所获得, 才有主观层面 上的体验。作为极具中国特色的时代词汇 “获得感” 直 译过来大多是 “The Gain of Sense” ，国外对此并没 有直接的研究。但是, 在国外的研究中有描述 “获得感” 的相关词汇概念, 诸如 “生活质量” (Life Quality)、 “生活满意度” (Life Satisfaction)、“主观幸福感” （Subjective We11-Being）等与获得感的意蕴内涵有 所相通的概念, 如生活质量往往指个体对所处生活状况 的主观感受, 主要由情感和认知两方面组成, 即个体在 实际生活中的情感体验和个体基于自身某种标准, 衡量 评价客观生活状况 ${ }^{[1]}$; 生活满意度是个体按照自己的标 准对自己一定时期内所处的生活领域如家庭、社会环境 等方面的生活质量的总体评价, 是一种主观的评价 ${ }^{[2]}$; 主观幸福感则是人们对自身生活满意度的一种认知评 价 ${ }^{[3]}$ 。这些与获得感相关的概念可以对获得感概念的界 定有所启发。

对于获得感的研究当前可以说是方兴未艾, 从已有 
的研究来看, 不同的学科对获得感的概念内涵的阐述非 常纷杂。在法学层面上认为, 人的获得感, 在法律上是 对人的生存发展权益的充分保障, 是对人的权利、尊严 与价值的制度保障, 正如黑格尔的法律体系中所言, 人 之所以为人的那种富有尊严感与获得感的人格是一切 法律所必备的精神和基本出发点 ${ }^{[4]}$ 。在社会学领域认为 人的获得感来源于个体通过与社会生活的相互作用, 在 社会群体中找到自己的定位与归属, 不断丰富完善自己, 在社会的大家庭中创造实现自身的价值, 为社会的存在 和延续创造条件 ${ }^{[5]}$ 。在哲学上对于获得感的探讨主要在 于人类自身对于物质财富的认可与获取以及对人的尊 严的关注与捍卫, 正所谓 “仓廪实而知礼节, 衣食足而 知荣辱”。在教育学上有从学校教育角度提出中小学教 育应让师生在学校生活中 “有更多获得感”, 要以 “人” 为目的, 学校教育才可能成全 “人” 的 “获得感”; 以及 从学生学习体验角度提出, 学生获得感主要是指学生学 习知识和能力的主观心理感受。心理学层面认为个体生 来就有对于食物的获得、保护与击积意识, 这个是在长 期的生物进化过程中, 动物与生俱来的一种保护巢穴与 获得与固积食物的本能。人们会无意识地主动去守护一 些东西, 在这个守护的过程中不断获得内心的快乐体验, 那些天生的奢徒就是因为获得本能过于强势, 获得感给 他们带来了强烈的心理刺激 ${ }^{[6]}$ 。美国的弗洛姆则认为, 个体只有不去占有其他东西, 也不渴望去占有, 心中就 会充满快乐, 他认为这是一种存在感, 一种并不一定追 逐拥有什么, 却内心足够丰富的一种意义上的获得感 ${ }^{[7]}$ 。

国内学者周海涛在对民办高校学生获得感的调查 与分析中指出 “学生获得感” 就是指学生在求学期间, 因学校提供的教育服务满足了学生学习和生活的需求, 获得了参与机会, 得到了认同, 并取得了一定成就的正 向综合心理感受; 何小芹从社会比较的角度探讨了相对 获得感, 也称为主观获得感, 是指个体或者群体通过与 参照对象比较而感知到自身处于有利地位, 进而体验到 愉快的一种主观认知和积极情绪体验; 董洪杰通过对 “获得感” 的结构探索, 认为中国人的获得感是个体对 获取自身需求满足的内容、实现途径与所需条件的认知 评价以及在此过程中的心理体验。

综合以上观点, 笔者认为, 获得感是指个体在社会 生活中, 对自身需要的满足而产生的积极的心理体验, 包含着情绪、认知、价值观和行为倾向等基本维度。

\section{3. 获得感的结构与测量}

对个体的主观体验的测量, 最初可以追溯到古希腊 时期的思想家柏拉图, 他在他的著作《理想国》一书中 测量出国王比独裁者拥有更多的幸福 ${ }^{[8]}$ 。柏拉图对于幸 福的测量方式虽然不能得以经过科学的严谨验证, 但是 他的思想激发了后人对于幸福感等主观的心理体验的 测量。英国学者边沁在 18 世纪提出了幸福是可以通过 快乐与否的强度、持续时间、真实性等几个要素衡量的 [9]。20世纪以来西方发达国家社会经济物质水平发展迅 速, “生活质量” 这一概念应运而生, 经济学家们将国民
收入作为基本要素衡量人民的生活质量水平。

作为一个新兴的学术词汇, 基于不同的研究目的与 研究传统, 目前各个学科都从不同的学科视角对获得感 进行了测量, 并且都在一定程度上取得了一定的研究成 果, 但是由于当前获得感的内涵结构不明, 使得测量指 标的选择旨趣迥异。

当前对于获得感的测量主要集中在一下两个方面： 一类是基于社会调查项目的测量研究。王浦劬和季程远 ( 2018) 以相对剥夺感为镜鉴, 并结合时空差别提出了 “纵向获得感”与 “横向获得感” , 调查我国人民 “获 得感” 的实际状况及其发展趋势。吕小康（2018）基于 2006 至 2015 年间的中国社会状况综合调查 (CSS) 数据 中的纵向比较的获得感测量模式, 将获得感归结为个人 发展感、社会安全感、社会公正感和政府工作满意度四 个维度, 并研究发现在此期间中国社会的获得感整体呈 现上升的趋势。文宏 ( 2018) 利用 CSGS 调研数据对我 国人民获得感进行时序比较，考察了人们经济获得感、 政治获得感与民生获得感三方面的时序差异。

另一类则是围绕不同群体研究对象的心理量表的 编制。何小芹等 ( 2017) 编制了《贫困大学生相对获得 感量表》从经济条件、家庭支持、人际关系、学校支持、 教师关怀、发展机会六方面对贫困大学生的相对获得感 进行现状调查。周海涛等 ( 2016) 则编制了包括认同程 度、满足状况、参与机会、成就水平四维度的《民办高 校学生获得感调查量表》。董洪杰 (2019) 对中国人获得 感的结构进行了研究并编制了包括获得体验、获得环境、 获得内容、获得途径和获得分享五个维度的《获得感量 表》。

在已有的测量方式中，研究者通常将所谓的客观指 标的变化 (如收入水平提升) 等同于个体获得感的变化, 忽略了个体在这中间的心理过程和心理体验。总体来看, 由于获得感的内涵概念不明, 在这些研究中获得感的概 念内涵主要基于研究者从不同学科背景出发的逻辑建 构。对于个体的主观感受一般采用自我报告的方式进行 测量, 即可以在问卷中描述某种行为特征, 让个体自我 判断是否符合自我获得感的真实情况。

\section{4. 获得感的影响因素}

\section{1. 人口统计学因素}

国外学者在研究青少年生活满意度的相关因素过 程中发现, 人口统计学变量诸如年龄、性别等变量与青 少年的生活满意度之间呈弱相关, 且这些变量对生活满 意度的预测作用不大 ${ }^{[10]}$ 。同样，对于获得感而言，男性 和女性在客观获得基础上的获得感没有明显差异, 但是 社会对男女角色有着不同定位和不同标准, 一般女性低 于男性般女性低于男性, 比男性更容易满足, 而男性往 往雄心勃勃容易压抑自我, 且年龄越大, 个人的获得感 更高

Neto 发现个体的社会经济地位对生活满意度影响 
较大 ${ }^{[11]}$, 这与 Huebner 的研究结果一致, 即, 社会经济 地位低的学生比社会经济地位高的学生报告的社会经 济地位低 ${ }^{[12]}$ 。不同于生活满意度的是, 我国学者为了解 贫困大学生相对获得感是否受性别、家庭类型、独生与 否等人口学变量的影响, 在性别、家庭类型以及独生与 否三个人口学变量上进行独立样本 $t$ 检验, 结果发现 相对获得感在性别、家庭单亲与否、独生子女与否上均 不显著。为进一步探寻影响贫困大学生相对获得感的因 素, 对学校支持 $(M=11.07)$ 、人际关系 $(M=9.73)$ 、家庭 支持 $(M=9.19)$ 、教师关怀 $(M=9.09)$ 、发展机会 $(M=8.15)$ 、 经济条件 $(M=5.32)$ 这六个维度进行描述统计分析结果 发现, 学校支持是贫困生相对获得感来源的重要方面, 这可能与贫困学生在学生生活的过程中, 师生关系、学 校管理等方面给与他们的支持, 让学生感受到自己被关 心与爱护, 并在此基础之上, 相信自己是有存在的尊严 与价值, 从而体验到较高的获得感。

\section{2. 经济因素}

经济因素对于个体主观体验的影响是多方面的, 目 前的研究中发现, 个人收入对个体的主观幸福感的影响 较弱, 即使是在高收入人群中, 他们确实比全国样本人 群感到幸福, 但是收入的影响在其中仍然很小 ${ }^{[13]}$ 。另一 方面, 有研究者从社会层面总体收入水平的变化角度切 入研究收入对于个体主观体验的影响, 发现社会整体经 济的大规模变化对个体的心理健康水平会产生影响, 尤 其是在经济衰退期影响社会民众的抑郁率 ${ }^{[14]}$ 。

大部分经济发达地区的居民平均获得感较高, 且 经济收入对获得感的影响显著收入对获得感的影响显 著, 我国的研究也发现, 东部与西部地区的获得感明 显高于中部, 东部经济发展水平显著高于中部和西部 地区, 这可能与东部地区相较于中西部地区较高水平 上的物质获得提升了居民的获得感, 而西部地区可能 得益于西部大开发等国家重大战略实施带来的政策红 利。这个研究结果可以是在情理之中, 经济发达地区 的整体社会服务、教育、医疗等社会福利水平通常比 不发达的贫困地区好, 故人们的获得感也相应增高。

另一方面家庭经济收入对获得感也有一定的影响, 家庭经济因素与居民获得感表现出显著的相关, 家庭收 入越高, 代表家庭在物质方面的获得越多, 由物质带来 的获得感也会越高。童年期有留守经历的个体在生活满 意度、幸福感、安全感等方面明显低于童年非留守经验 的个体 ${ }^{[15]}$, 这其中主要影响因素还是家庭的经济收入。 这可能因为经济不富裕家庭学生一般较为自卑, 较少有 机会参与各种活动, 不能产生获得体验。

\section{3. 家庭因素}

家庭是个体融入社会时的最初单位, 在个体身心发 展中承担了不可忽视的作用。自上个世纪六七十年代以 来, 国外学者就针对家庭因素展开了一些列广泛的研究。
Glickman 根据家庭成员的社会交往风格、家庭关系 结构等方面认为适当型和最佳型家庭为健康家庭, 而极 端性的家庭会造成家庭成员的社会适应障碍等影响心 理健康水平的问题出现 ${ }^{[16]}$ 。Grossman 和 Rowat 在研究 家庭父母的婚姻与青少年的生活满意度的关系中也发 现, 父母的离婚、分居以及再婚都会造成青少年的生活 满意度、幸福感有所下降 ${ }^{[17]}$ 。个体的身心健康发展不仅 受到来自父母的家庭因素影响, 家庭中的其他成员也会 对他们产生复杂的影响, 如兄弟姐妹的关系, 这种因素 的影响在他们的青春期显得尤为突出, 复杂的社会情感 和认知发生变化对青少年的影响时巨大的, 这其中女生 相对男生影响更大。

家庭因素另一个方面影响个体身心发展的在于父 母的教养方式, Suldo 和 Huebner 发现权威型父母的教 养方式与青少年的生活满意度呈现显著的正相关, 这可 能因为权威性的父母更多的介入参与到了孩子的生活 中，让他们感受到了更多的家庭支持与参与有关 ${ }^{[18]}$ 。 Rask 从对青少年的研究中发现, 他们的满意感或不幸福 的感觉与他们所体会到的家庭气氛相关。家庭的稳定、 成员间的相互关怀、没有明显的家庭矛盾是青少年总体 满意度的预期因素 ${ }^{[19]}$ 。但是 Shek 在一系列针对中国经 济弱势青少年的研究中, 研究了感知父母行为、父母控 制过程、亲子关系质量、家庭功能与青少年心理健康、 药物滥用和违法行为之间的关系。结果显示, 经济弱势 青少年的生活满意度水平较低 ${ }^{[20]}$; 我国学者调查贫困大 学生相对获得感的时候发现, 在家庭给与的情感支持方 面, 以贫困生的相对获得感在家庭支持方面高于非贫困 生，但是差异未达到显著水平。虽然家庭经济条件存在 差异, 但是贫困生从家庭方面获得的情感支持并不会比 非贫困生少。

上述相关研究都从不同角度强调了家庭变量在个 体身体发展中的作用, 如家庭结构、父母教养方式、父 母情感等家庭相关的因素, 这些家庭因素对于个体获得 感的实现是至关重要的。

\section{4. 情绪智力}

情绪智力联结了个体认知和情绪两个重要的心理 过程, 是促进个体心理健康的一个重要能力 ${ }^{[21]}$ 。目前许 多研究倾向于将情绪智力引入到个体的谁会适应、心理 调适、主观幸福感、生活满意度的研究, 尤其是在个体 的职业、社会生活、学业等领域。情绪智力在很大程度 上左右着生活满意度, 人的情绪智力越高, 积极情绪体 验就越多, 从而使个体更可能有着较高的生活满意度。

情绪智力中的情绪管理能力也会对个体的获得感 产生一定的影响。我国学者周海涛 (2016) 为深入探讨 情绪管理、学习力和能力发展对学生获得感的影响, 将 获得感作为效标变量加入因变量, 将情绪管理、学习力、 能力发展作为预测变量加入自变量, 通过回归分析发现 青少年情绪管理能力对获得感的有影响。

情绪智力的概念是多元的, 包括情绪的自控能力、 管理能力、表达能力等方面, 其对个体保持心理健康的 
作用不言而喻, 后期演技可以从个体的情绪智力作为切 入点, 对获得感展开进一步深入的研究。

\section{5. 人格因素}

自美国学者 Tellegen 等人在双生子爬梯实验中发 现人格中的气质因素对儿童的情感体验产生了重要的 作用以来, 学术界将人格的各个因素与个体内部心理体 验的研究推向了高潮。DeNeve 研究了一项大五人格风格 与生活满意度之间的元分析, 从中发现人格维度中的外 倾性与宜人性可以预测个体积极的性感，其中严谨行与 生活满意度的相关最高 ${ }^{[22]}$ 。Tucker 在 1998 年的研究也 进一步验证了 DeNeve 的研究结果, 他发现快乐的人更 倾向于积极地解释生活中遇到的事件相比于不快乐的 人来说。Schmutte 和 Ryff 的研究发现, 外向性、尽责 性和低神经质的个体更倾向于自我接受、掌控生活。

正如人们经常被问到的问题: “什么样的人可能会 幸福?”、“什么样的人又可能会经常感受到获得感? ”, 是否存在某些人格因素始终与获得感相关, 获得感本身 是否可以被认为是一种人格变量? 这些问题有待在后续 的研究中进一步展开深入。

\section{5. 展望}

获得感诞生于特殊的时代, 是当前人们群众对于美 好生活向往的内涵体现, 更是一个极具中国时代色彩的 新词。社会各界对于获得感的关注可以说是到了一个前 所未有的高度, 也是社会心理学在改革浪潮中的蓬勃发 展的体现。

获得感毫无疑问是一种带有浓厚个人主观色彩的 心理体验, 不同的人有不同的获得感。贫穷的乞正可以 因为得到一个硬币而产生获得感, 但是腰细万贯的富翁 却未必比乞正有更高的获得感。因此, 对获得感的深人 研究是了解个人满足美好生活向往的重要依据, 也是调 节个人生活与心理健康的一个重要手段。今后的研究可 以深人探讨以下几方面的问题: (1) 进一步探讨影响获 得感的其他因素, 如人格特质、归因方式、人际关系、家 庭文化背景、家庭结构等是如何影响个人获得感的, 以 及它们对个人获得感影响作用的不同权重; (2) 探讨不 同群体 (如青少年学生、军人、党员、下岗职工、社会高 收人者等) 的获得感; (3) 进一步将获得感与幸福感、满 足感、成就感等心理概念进行辨析, 建构获得感的相关 理论模型; (4) 研究方法的创新, 近年来国内外运用较多 的研究方法是自我报告法, 除此之外, 国外一些学者也 在尝试着运用实验的方法进行研究, 这也是我们今后努 力的一个方向。

\section{REFERENCES}

[1] Henrich, G., \& Herschbach, P. (2000). Questions on life satisfaction (flzm) - a short questionnaire for assessing subjective quality of life. European Journal of Psychological Assessment, 16(3), 150-159.

[2] Pavot, W., \& Diener, E. (2008). The satisfaction with life scale and the emerging construct of life satisfaction. Journal of Positive Psychology, 3(2), 137-152.

[3] Diener, E., Diener, M., \& Diener, C. (1995). Factors predicting the subjective well-being of nations. Journal of Personality \& Social Psychology, 69(5), 851-864.

[4] Georg Wilhelm Fredrich. (1991). Hegel: elements of the philosophy of right. German History, 11(2), 243-244.

[5] Festinger, L. A.. (1954). A theory of social comparison processes. Human Relations, 7(2), 117--140.

[6] McDougall, W. (1924). Outline of psychology. journal of neurology \& psychopathology, s1-5(18), 184.

[7] Fromm, E. (1980). Beyond the Chains of Illusion: My Encounter with Marx and Freud.

[8] Mannheim, K., \& Wirth, L. (1938). Ideology and utopia: An introduction to the sociology of knowledge. The American Historical Review, 43(4).

[9] Morgan, E. V., Bentham, J., \& Stark, W. (1953). Jeremy Bentham's Economic Writings. Vol. III.. The Economic History Review, 6(2).

[10] Gilman, R. , \& Huebner, S. . (2003). A review of life satisfaction research with children and adolescents. School Psychology Quarterly, 18(2), 192-205.

[11] Félix Neto. (1993). The satisfaction with life scale: psychometrics properties in an adolescent sample. Journal of Youth and Adolescence, 22(2), 125-134.

[12] Ash, C., \& Huebner, E. S. (2001). Environmental Events and Life Satisfaction Reports of Adolescents. School Psychology International, 22(3), 320.

[13] Diener, E., Horwitz, J., \& Emmons, R. A. (1985). Happiness of the very wealthy. Social Indicators Research, 16(3), 263-274.

[15] Zhe, L. I. (2009). The Situation of PTSR,Social Support and Sense of Security One Month after the Wenchuan Earthquake and the Relationship Among Them. Journal of Southwest University.

[16] Glickman, H. S. (1977). Book Review: No Single Thread: Psychological Health in Family Systems. Social casework, 58(2), 123-124.

[17] Grossman, M., \& Rowat, K. M. . (1995). Parental relationships, coping strategies, received support, and wellbeing in adolescents of separated or divorced and married parents. Research in nursing \& health, 18(3), 249-261. 
[18] Suldo, S. M., \& Huebner, E. S. (2004). Does life satisfaction moderate the effects of stressful life events on psychopathological behavior during adolescence?. School Psychology Quarterly, 19(2), 93-105.

[19] Rask, K., Pivi Åstedt-Kurki, Paavilainen, E., \& Laippala, P. (2003). Adolescent subjective well-being and family dynamics. Scandinavian Journal of Caring Sciences.

[20] Daniel T. L. Shek. (2003). Chinese adolescents and their parents' views on a happy family: implications for family therapy. family therapy the journal of the california graduate school of.

[21] Jacobs, M., Snow, J., Geraci, M., Vythilingam, M., Blair, R. J., Charney, D. S., ... \& Blair, K. (2008). Association between level of emotional intelligence and severity of anxiety in generalized social phobia. Journal of Anxiety Disorders, 22(8), 1487-1495.

[22] Deneve, K. M., \& Cooper, H. . (1998). The happy personality: a meta-analysis of 137 personality traits and subjective well-being. Psychological Bulletin, 124(2), 197229.

[23] Lyubomirsky, Sonja, \& Tucker, Kari L KL. (1998). Implications of individual differences in subjective happiness for perceiving, interpreting, and thinking about life events 1 . motivation \& emotion, 22(2), 155-186.

[24] Schmutte, Pamela, S., Ryff, Carol, \& D. (1997). Personality and well-being: reexamining methods and meanings. Journal of Personality \& Social Psychology. 